\title{
A Study on Formation Process of Secondary Upsetting Defect in Electric Upsetting and Optimization of Processing Parameters Based on Multi-Field Coupling FEM
}

\author{
Guo-zheng Quan ${ }^{a, b^{*}}$, Zhen-yu Zou', Zhi-hua Zhang ${ }^{b}, J_{i a}$ Pan $^{b}$ \\ ${ }^{a}$ State Key Laboratory of Mechanical Transmission, School of Material Science and Engineering, \\ Chongqing University, Chongqing 400044, China \\ ${ }^{b}$ State Key Laboratory of Materials Processing and Die \& Mould Technology, Huazhong University of \\ Science and Technology, Hubei 430074, China
}

Received: November 10, 2015; Revised: March 1, 2016; Accepted: June 13, 2016

\begin{abstract}
The electric upsetting process is an excellent electrically assisted preforming technique with electrical-thermal-mechanical multi-field coupling characteristic. The secondary upsetting defect in electric upsetting with inappropriate parameters combinations could result in folds in subsequent forging process. In order to analyze the formation process of the secondary upsetting defect, the finite element model of electric upsetting was constructed based on the multi-field coupling solver platform, MSC. Marc. It is concluded through the numerical simulation with inappropriate parameters combination that the main factor causing the secondary upsetting defect is the large temperature decrease of the deformed portion after the formation of drum-shape in the preliminary stage of electric upsetting. Subsequently, the secondary upsetting defect has been successfully avoided via a three-stage current mode. Finally, a novel optimization method based on close-cycle control of current in numerical simulation was established by developing a current subroutine in MSC.Marc, which was proved to be effective for the elimination of the secondary upsetting defect.
\end{abstract}

Keywords: Multi-field coupling, Electric upsetting, Defects, Numerical simulation

\section{Introduction}

High-alloy metals are in vogue for the manufacture of lightweight structures such as airplane frameworks and engine parts on account of their high specific strength and excellent heat resistance. Nevertheless, most highalloy metals exhibit poor workability in traditional plastic forming process ${ }^{1}$ because the effective temperature range of plastic deformation for high-alloy metals is very narrow, which has blocked the widespread application of high-alloy metals. Therefore, an effective low-speed forming process kept at elevated temperatures for high-alloy metals is essential at present. Electrically assisted plastic forming, as a non-traditional processing technique, is able to enhance metals' formability during deformation ${ }^{1,2}$. The simultaneous application of electricity during plastic deformation ensures that the deformation temperature is above recrystallization temperature, which therefore lowers the yield strength and increases the ductility for high-alloy metals. Meanwhile, the hydrostatic system provides an approximate isostatic pressing process, which lowers metals' work hardening rate and increases its microstructural stability.

Engine valves are important parts of an engine that work as controllers of air intake and exhaust in a working cycle of engine. The engine valve products are typical long rod parts with thin flange on one end. In the past, the preforming process of the engine valve products is hot extrusion, which is inefficient. In order to enhance productivity, the electric upsetting is adopted as a more intensive utilized process

* e-mail: quangz3000@sina.com to preform exhaust valves. The forming steps of an engine valve are shown in Figure 1.

Electric upsetting is an excellent electrically assisted preforming process capable of creating a local enlarged diameter on a bar, and the preformed workpiece can then be forged without further heating. It has been widely adopted to the manufacture of engine valves for automobiles, ships and aircrafts due to its high productivity and low cost. The schematic drawing of electric upsetting process is shown in Figure 2. A cold bar stock is placed between the anvil and the upsetting cylinders. A low-voltage, high-amperage, adjustable direct current is passed into the portion of the bar between the anvil and the clamping electrode. As the galvanic portion of material has been heated (by self-resistance and contact resistance) and becomes plastic, the upsetting cylinder provides an adjustable axial pressure to compress the bar and enlarge it at the hot end and create a ball against the anvil. Meanwhile, the anvil cylinder moves back with an adjustable speed to ensure a steady deformation process.

In recent years, the widespread application of finite element method has made great difference in hot forming field. In earlier times, Eggert, et al. and Nied, et al. developed a finite element model to simulate the resistance upset welding process and to study the role and sensitivity of major process parameters ${ }^{3,4}$. Kim, et al. predicted the optimum conditions of electric upsetting process through simulation on "QForm" platform of the engine valve with a diameter of $73 \mathrm{~mm}^{5}$. Previous studies contribute to the development of a thermo-mechanical numerical model for electric upsetting. Nowadays, it is commonly believed that several commercial platforms involving nonlinear finite element analysis, including 


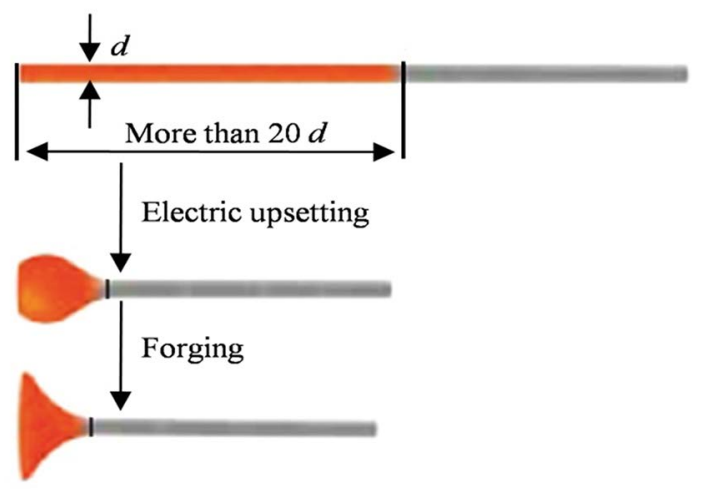

Figure 1: Forming steps for an engine valve.

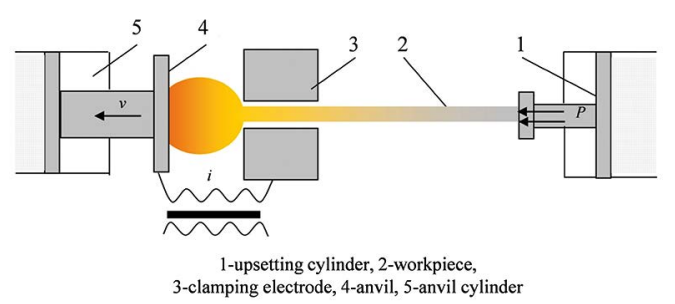

Figure 2: Schematic drawing of electric upsetting process.

QForm, MSC.Marc, Ansys, etc. are available in numerical simulation of electric upsetting process.

Generally, the preformed shape by electric upsetting should be controlled in a reasonable range, so as to ensure the forming quality of engine valves in final forming process. The qualified shapes by electric upsetting are shown in Figure 3, in which the A-type shape with nearspherical head is the ideal preformed shape. In practical production, the preformed shape by electric upsetting should fall in between B-type shape and C-type shape. Therefore, the optimization of processing parameters to obtain the ideal A-type preformed shape by electric upsetting is of great significance. However, more processing parameters including clamping length, upsetting current, astern speed of anvil and upsetting pressure are involved in electric upsetting process, in comparison with traditional forging processes. An inappropriate parameters combination in electric upsetting process could result in unqualified shape or even lead to defects like external waviness and secondary upsetting defect, as shown in Figure 4a-b, respectively. Minor external waviness defect on the preformed bar can be eliminate in final forming process. However, the secondary upsetting defect on the preformed bar is the major factor lead to rejects since the groove between two drum-shapes is very deep, which results in folds in subsequent forging process. Thus the study on secondary upsetting defect in electric upsetting is of great significance. Over the past few decades, efforts have been made for the optimization of processing parameters to obtain satisfactory shape in electric upsetting process. Wang, et al. summarized the influences of processing parameters on the forming quality for electric upsetting by theoretical analysis and

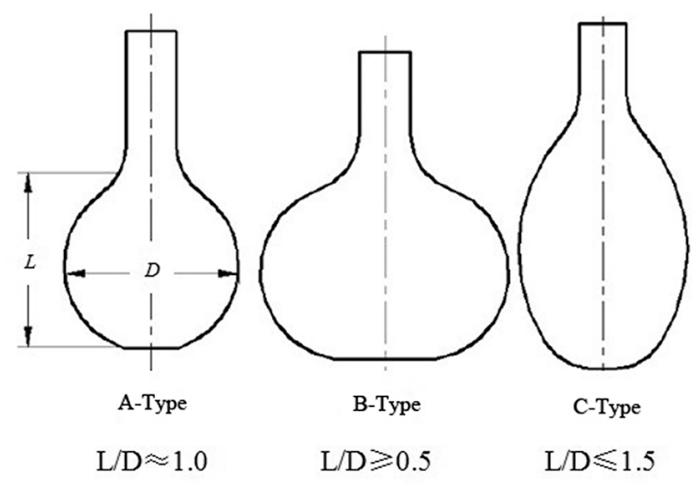

Figure 3: The qualified shapes by electric upsetting.

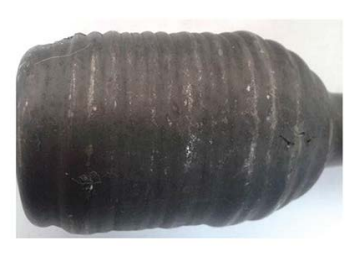

(a)

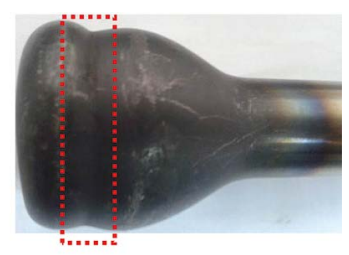

(b)
Figure 4: Defects in electric upsetting: (a)external waviness, (b) secondary upsetting defect.

practical experiment, and developed a microcomputer control system for electric upsetting to eliminate the defects and improve the forming quality ${ }^{6}$. Liu, et al analyzed the main processing parameters, and introduced a new computer-controlled electrical upsetting system ${ }^{7}$. The results have demonstrated that the optimized processing parameters can obtain qualified shape and improve the forming quality in electric upsetting process. However, few investigations were focus on the solution of secondary upsetting defect in electric upsetting process.

In order to study the forming characteristics and optimize the processing parameters of electric upsetting process for an engine valve with $3 \mathrm{Cr} 20 \mathrm{Ni} 10 \mathrm{~W} 2$ heat-resisting alloy in marine diesel engine, a 2D finite element model with implicit method for electric upsetting was constructed based on the commercial software MSC.Marc, in which the electrical-thermal-mechanical multi-field coupling method was adopted. The numerical simulation for electric upsetting under inappropriate processing parameters combination was performed, and the typical secondary upsetting defect caused by inappropriate parameters was analyzed. The results have shown that the secondary upsetting defect can be avoided by the adjustment of upsetting current. A three-stage loading mode of direct current was introduced to prevent the workpiece from the secondary upsetting defect. Finally, a new optimization method was proposed for electric upsetting process by introducing a self-adaptive boundary current subroutine which was based on the perspective of stability control of deformation temperature in numerical simulation. The optimized current parameter output from FE software could play a role for practical production, since it has been proved to be effective in an electric upsetting experiment. 


\section{Finite element model of electric upsetting}

\subsection{Heating analysis of electric upsetting}

During the electric upsetting process, deformation is accompanied by heating. Thus the temperature distribution on billet makes great difference on the final shape and forming quality of the workpiece. The temperature rise of the billet is mainly due to heat generated by joule effect, by plastic deformation and by friction between billet and anvils, while the heat loss of the billet is attributed to the heat conduction between billet and anvils, the heat convection and heat radiation with the surroundings.

The heat generated by joule effect can be divided into two parts. One is joule heat generated by volume resistance of billet, which can be expressed by joule's law in Equation 1. The other is joule heat generated by contact resistance between billet and anvils, part of which is conducted to billet. As the joule heat in contact surface is conducted to billet and anvils proportionally to their heat conductivity, the part of heat conducted to billet can be expressed by Equation 2,

$$
\begin{gathered}
Q_{I V}=I^{2} R_{v} \\
Q_{I C}=\frac{k_{b}}{k_{b}+k_{a}} I^{2} R_{c}
\end{gathered}
$$

where $Q_{I V}$ is the heat generated by the volume resistance of billet; $Q_{I C}$ is the part of joule heat in contact surface conducted to billet; $I$ is current intensity; $R_{V}$ is the volume resistance of billet; $R_{C}$ is the contact resistance between billet and anvils; $k_{b}$ and $k_{a}$ is the heat conductivity of billet and anvils, respectively.

The heat generated by plastic deformation can be calculated by Equation 3,

$$
Q_{p}=\beta \bar{\sigma} \dot{\bar{\varepsilon}}
$$

where $Q_{P}$ is the heat generated by plastic deformation; $\beta$ is the conversion coefficient between deformation work and heat; $\bar{\sigma}$ is equivalent stress; $\dot{\bar{\varepsilon}}$ is equivalent strain rate

The heat generated by friction between billet and anvils can be expressed by Equation 4,

$$
Q_{f}=\int \tau|\Delta v| d \Gamma
$$

where $Q_{f}$ is heat generated by friction; $\tau$ is friction; $\Delta v$ is relative velocity.

On the contrary, the heat loss of the billet by heat conduction to the anvils, heat convection and heat radiation with the surroundings can be expressed by Equation 5 to Equation 7 respectively.

$$
\begin{gathered}
Q_{c}=h_{c} A_{c}\left(T_{b}-T_{a}\right) \\
Q_{S}=h_{S} A_{S}\left(T_{B}-T_{S}\right) \\
Q_{r}=\varepsilon A_{r} \sigma_{0}\left(T_{b}^{4}-T_{S}^{4}\right)
\end{gathered}
$$

where $Q_{c}$ is heat loss of the billet by heat conduction to the anvils; $Q_{S}$ is the heat loss by heat convection with the surroundings; $Q_{r}$ is heat loss by heat radiation with the surroundings; $h_{c}$ is the heat conductivity of contact surface between billet and anvils; $h_{S}$ is heat conductivity between billet and the surroundings; $A_{c}$ is the area of contact surface between billet and anvils; $A_{S}$ is the area of billet exposed to the surroundings; $A_{r}$ is the heat radiation area on the billet; $T_{b}, T_{a}$ and $T_{s}$ is temperature of billet, anvils and the surroundings, respectively.

As a result, the heat of the billet can be expressed by Equation 8,

$Q=Q_{I V}+Q_{I C}+Q_{f}+Q_{p}-Q_{c}-Q_{S}-Q_{r}$

\subsection{Theory of rigid-plastic finite element method}

The rigid-plastic finite element method is suitable for the deformation analysis of various metal forming processes with large deformation. The accuracy of the method is usually reasonably good for most practical purposes and the computation procedure is compact and fast, which have promoted the widespread of this method in the analysis metal forming problems. The deformation process for rigidplastic materials is associated with boundary-value problems where both the stress and velocity field solutions satisfy the equilibrium equations and the constitutive equations in the domain, and the prescribed velocity and tractions on the boundary. The duality of the boundary value problem and the variation problem can be seen clearly by considering the construction of the function expressed in Equation $9^{8}$,

$$
\pi=\int_{V} \bar{\sigma} \dot{\bar{\varepsilon}} d V-\int_{S F} F_{i} u_{i} d S
$$

where $\pi$ is a function of the total energy and work, $\bar{\sigma}$ the effective stress, $\dot{\bar{\varepsilon}}$ the effective strain rate, $F_{i}$ represents the surface tractions and $u_{i}$ is the velocity component.

The incompressibility constraint on admissible velocity fields in Equation 9 can be removed by using the penalized form of the incompressibility. Hence, the variational form used as the basis for finite element discretization is given by Equation 10,

$$
\delta \pi=\int_{V} \bar{\sigma} \delta \dot{\bar{\varepsilon}} d V+K \int_{V} \dot{\varepsilon}_{v} \delta \dot{\varepsilon}_{v} d V-\int_{S F} F_{i} \delta u_{i} d S
$$

where $\ddot{a} \dot{\bar{\varepsilon}}$ and $\ddot{a} \dot{\varepsilon}_{v}$ are the variations in strain rate and volumetric strain rate, respectively, and $K$ is a penalty constant having a very large positive value. Equation 9 or 10 represents the basic equation for the finite element formulation.

\subsection{Electrical-thermal-mechanical coupling analysis}

For electrical-thermal-mechanical problems, it is difficult to develop a finite element analysis model due to the significant interaction of temperature field, electric field and mechanic field. Thus it is of great significance to capture the coupling relationship between the three fields. Figure 5 shows the schematic diagram of coupling relationship between temperature field, electric field and mechanic field. It can easily be found in Figure 5 that the coupling relationship 


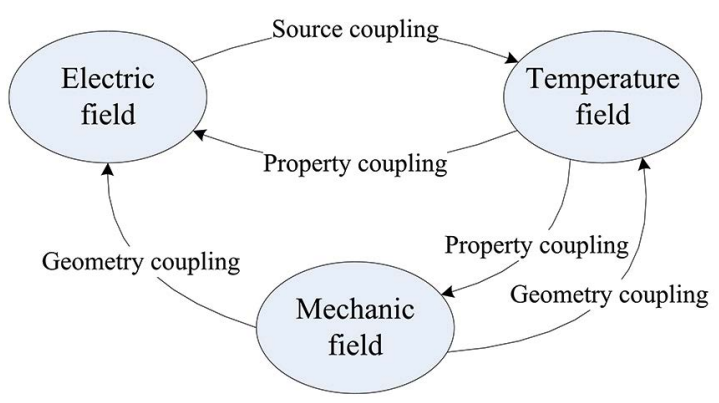

Figure 5: Schematic diagram of coupling relationship between temperature field, electric field and mechanic field.

between electric field and temperature field as well as the coupling relationship between temperature field and mechanic field is interacted, while the electric field is unidirectionally coupled with the mechanic field. As a result, the electricalthermal-mechanical coupling analysis (Joule-mechanical) can be regarded as a combination of electrical-thermal analysis (Joule heating) and thermal-mechanical analysis. Therefore, it is vital to implement a coupling procedure between electrical-thermal and thermal-mechanical modules to capture this physical interaction and produce a realistic finite element analysis model ${ }^{9,10}$. The multi-field coupling analysis is handled using a staggered solution procedure. Through this approach, firstly the electric problem is solved for the nodal voltages. Secondly, the thermal problem is solved to achieve the nodal temperatures. The mechanical problem is solved at last for the nodal displacements. The matrix equations governing the electrical, thermal and mechanical problems are expressed as Equation $11^{11}$,

$$
\left\{\begin{array}{l}
K^{E}(T) V=I \\
C^{T}(T) \dot{T}+K^{T}(T) T=Q+Q^{E}+Q^{I}+Q^{F} \\
M \ddot{u}+D \ddot{u}+K^{M}(T, u, t) u=F+F^{T}
\end{array}\right.
$$

where $V$ is the nodal voltage vector; $T$ is the nodal temperature vector; $u$ is the nodal displacement vector; $K^{E}(T)$ is the temperature-dependent electrical conductivity matrix; $I$ is the nodal current vector; $C^{T}(T)$ is the temperature-dependent heat capacity matrix; $K^{T}(T)$ is the temperature-dependent thermal conductivity matrix; $Q$ is the flux vector; $Q^{E}$ is the heat generation due to electrical flow vector; $Q^{I}$ is the heat generation due to inelastic deformation vector; $Q^{F}$ is the heat generation due to friction vector; $M$ is the mass matrix; $D$ is the damping matrix; $K^{M}(T, u, t)$ is the temperature, deformation and time-dependent stiffness matrix; $F$ is the externally applied force vector; $F^{T}$ is the force due to thermal strain vector.

\subsection{Finite element model of electric upsetting}

The $2 \mathrm{D}$ finite element model of electric upsetting process was constructed through the commercial software MSC. Marc based on the electrical-thermal-mechanical multi-field coupling method, as shown in Figure 6. The half-symmetry model was adopted in current work since the electric upsetting system exhibits axial symmetry. A lower-order, four-node quadrilateral finite element formulation were selected for all bodies of this assembly. The finite-element mesh was generated automatically for the initial bar shape and it could be automatically regenerated at each time increment when the bar starts to deform. The anvil, as well as the clamping electrode, was set as rigid objects, while the billet was set as plastic object. Usually, the anvil and the clamping electrode are treated as the boundaries of current flow. Therefore, the heat transfers between clamping electrode and bar, between anvil and bar, and between all the objects and environment were all taken into consideration.

Considering the great effect of contact resistance in the preliminary stage of electric upsetting process, a typical contact resistance model which is closely dependent on contact surface temperature and contact pressure was adopted in current work, as expressed in Equation 12.

$$
\rho=1 /\left(a_{1} T+a_{2}\right)
$$

where $\tilde{n}$ is the resistivity of contact resistance; $T$ is the heating temperature; $a_{1}$ and $a_{2}$ are the constants dependent on contact pressure, here their values are 0.87 and 132 respectively.

The material physical properties of billet, anvil and clamping electrode are listed in Table $1^{12,13}$. The adopted stress-strain data of billet material, 3Cr20Ni10W2 heat-resistant alloy, which was obtained from a series of isothermal compressions with a height reduction of $60 \%$ over a temperature range of $930 \sim 1130{ }^{\circ} \mathrm{C}$ and a strain rate range of $0.01 \sim 10 \mathrm{~s}^{-1}$ on Gleeble-1500 simulator are shown in Figure 7. In addition, the physical parameters such as friction coefficient, heat generation conversion factor, heat transfer coefficient, etc. in the finite element model are listed in Table $2^{13}$.

\section{Results and discussions}

\subsection{Analysis on formation process of the secondary upsetting defect by numerical simulation}

The workpiece with typical secondary upsetting defect preformed by electric upsetting process for $3 \mathrm{Cr} 20 \mathrm{Ni} 10 \mathrm{~W} 2$ heat-resistant alloy is shown in Figure 4b. It is obviously found that there are two drum-shapes on the deformed region of the workpiece, and the groove between the two drum-shapes is very deep, which may lead to folds in subsequent forging process. In order to study the formation process of secondary upsetting defect, a numerical simulation of electric upsetting process with inappropriate processing parameters combination was conducted. The original processing parameters adopted in current work are listed in Table $3^{13}$.

The numerical simulation result for formation process of secondary upsetting defect is shown in Figure 8, in which the color map on the workpiece represents temperature distribution. It is similar to the formation process of secondary upsetting defect in actual production shown in Figure 9. In the preliminary stage of electric upsetting, the galvanic portion of the bar between the anvil and the clamping electrode was heated rapidly to the forging temperature and experienced severe deformation under the axial upsetting pressure. As a result, the severe radial flow of materials induced a rapid increase in diameter and a rapidly decrease in length of the galvanic area, forming a large slope on the drumshape, as shown in Figure 8a. However, the plastic deformation of the galvanic area, as well as the decrease of current density due 


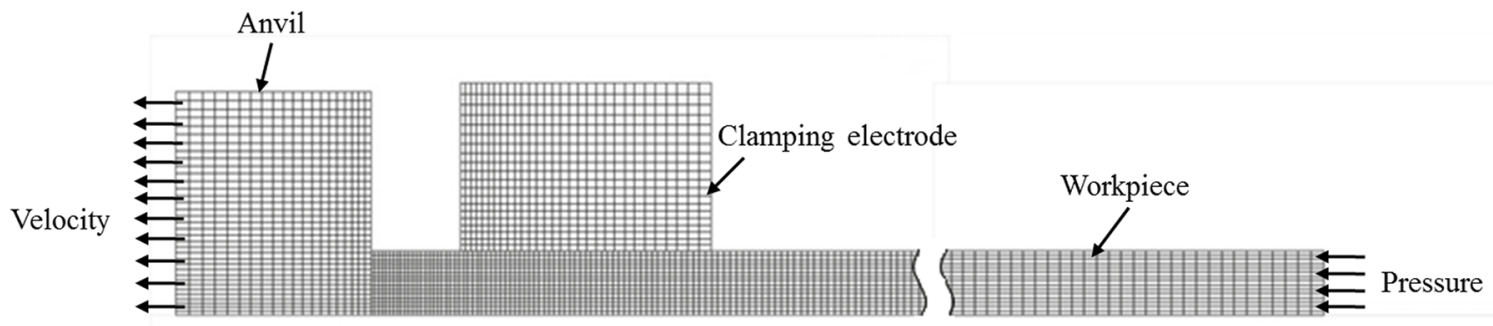

Figure 6: The finite element analytical model of electric upsetting process.

Table 1: Physical properties of the materials.

\begin{tabular}{lcccccccc}
\hline & & \multicolumn{7}{c}{ Temperature $\left({ }^{\circ} \mathrm{C}\right)$} \\
& & 27 & 227 & 427 & 627 & 827 & 927 & 1127 \\
\hline Billet & $\theta(\mu \Omega \cdot \mathrm{m})$ & 1.150 & 1.176 & 1.198 & 1.230 & 1.275 & 1.295 & 1.320 \\
& $\mathrm{C}\left(\mathrm{J} /\left(\mathrm{g} \cdot{ }^{\circ} \mathrm{C}\right)\right)$ & 0.491 & 0.495 & 0.5255 & 0.6008 & 0.666 & 0.759 & 0.6733 \\
& $\lambda\left(\mathrm{W} /\left(\mathrm{m} \cdot{ }^{\circ} \mathrm{C}\right)\right)$ & 9.6 & 10.4 & 14.3 & 17.9 & 21.1 & 22.7 & 26.0 \\
& $\rho\left(\mathrm{Kg} / \mathrm{m}^{3}\right)$ & & & & 8512 & & & 35.64 \\
Anvil and Clamping electrode & $\theta(\mu \Omega \cdot \mathrm{m})$ & 5.78 & 11.39 & 17.65 & 23.23 & 29.83 & - & 0.320 \\
& $\mathrm{C}\left(\mathrm{J} /\left(\mathrm{g} \cdot{ }^{\circ} \mathrm{C}\right)\right)$ & 0.250 & 0.265 & 0.274 & 0.291 & 0.300 & -26.0 & 26.0 \\
& $\lambda\left(\mathrm{W} /\left(\mathrm{m} \cdot{ }^{\circ} \mathrm{C}\right)\right)$ & 24.6 & 25.0 & 25.5 & 26.0 & 26.0 & 26.0 \\
& $\rho\left(\mathrm{Kg} / \mathrm{m}^{3}\right)$ & & & & 10200 & & &
\end{tabular}

Note: $\theta$ - resistivity, $\mathrm{C}$ - specific heat, $\lambda$ - heat conductivity, $\rho$ - Density

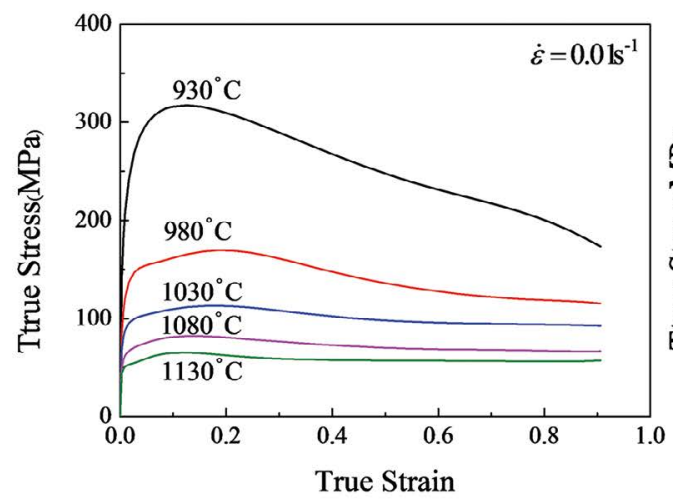

(a)

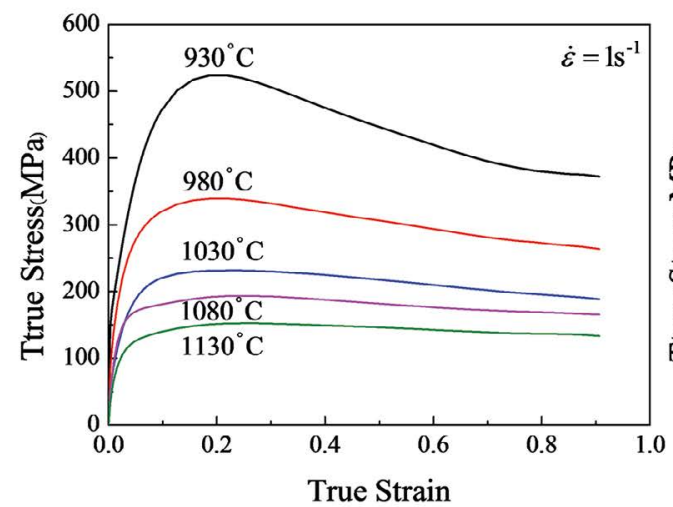

(c)

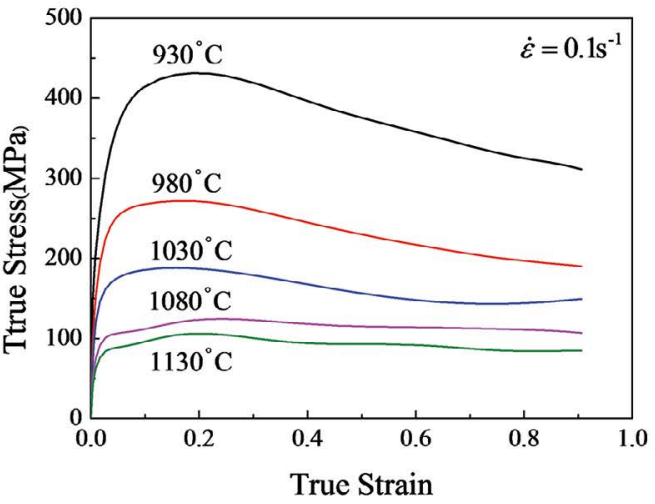

(b)

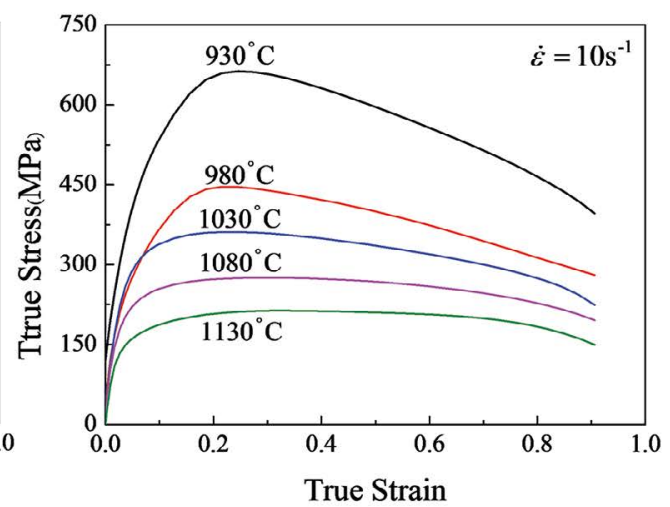

(d)

Figure 7: The true stress-strain curves of $3 \mathrm{Cr} 20 \mathrm{Ni10W} 2$ alloy at different temperatures and strain rates (a) $0.01 \mathrm{~s}-1,930 \sim 1130{ }^{\circ} \mathrm{C}$; (b) 0.1 $\mathrm{s}-1,930 \sim 1130{ }^{\circ} \mathrm{C}$; (c) $1 \mathrm{~s}-1,930 \sim 1130{ }^{\circ} \mathrm{C}$; (d) $10 \mathrm{~s}-1,930 \sim 1130{ }^{\circ} \mathrm{C}$. 
Table 2: Physical parameters of finite element simulation.

\begin{tabular}{|c|c|c|}
\hline Physical parameter & & Value \\
\hline $\begin{array}{l}\text { Initial temperature of billet, anvil and clamping electrode } \\
\left({ }^{\circ} \mathrm{C}\right)\end{array}$ & & 20 \\
\hline Environment temperature $\left({ }^{\circ} \mathrm{C}\right)$ & & 20 \\
\hline Friction coefficient & & 0.3 \\
\hline Heat generation conversion factor & & 0.9 \\
\hline Heat transfer coefficient & between billet and anvil & 20 \\
\hline$\left(\mathrm{N} /\left(\mathrm{s} \cdot \mathrm{mm} \cdot{ }^{\circ} \mathrm{C}\right)\right)$ & between billet and environment & 0.02 \\
\hline
\end{tabular}

Table 3: Original processing parameters of the electric upsetting process.

\begin{tabular}{ll}
\hline Processing parameter & Value \\
\hline Upsetting current $(\mathrm{A})$ & 8042 \\
Clamping length $(\mathrm{mm})$ & 16 \\
Astern speed of anvil $(\mathrm{mm} / \mathrm{s})$ & 0.5 \\
Upsetting pressure $(\mathrm{MPa})$ & 225 \\
\hline
\end{tabular}

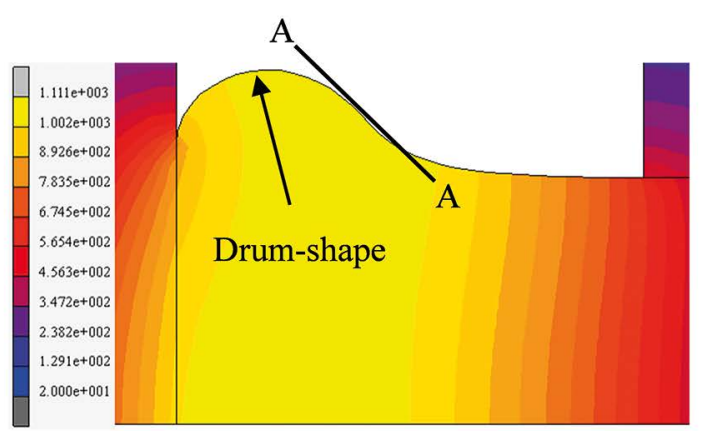

(a)

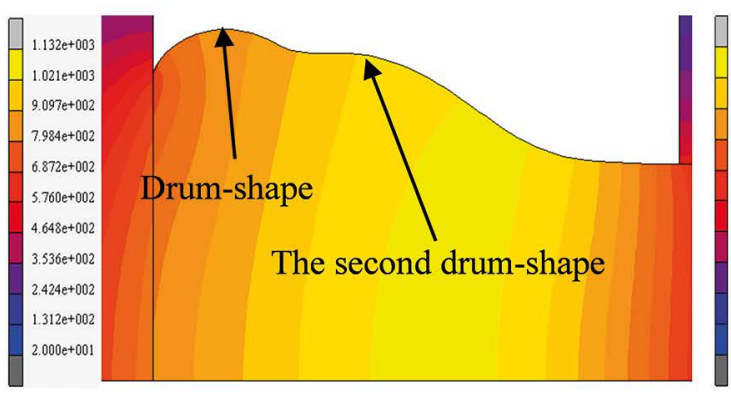

(c)

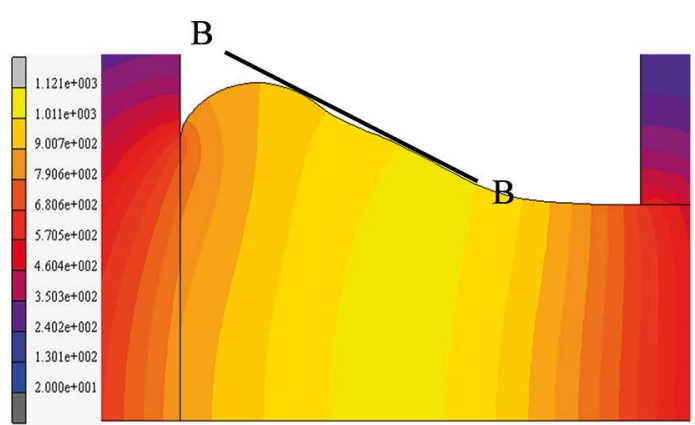

(b)

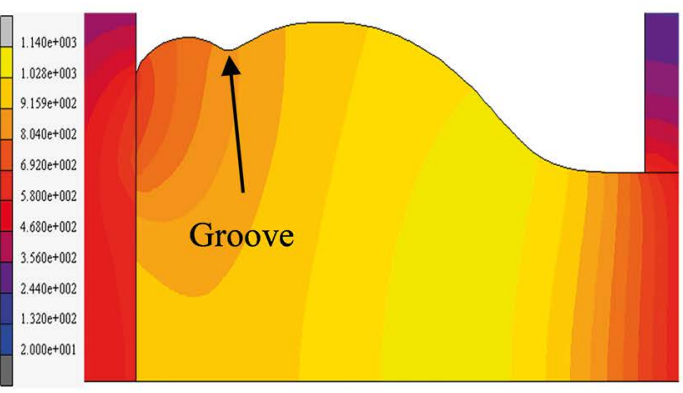

(d)

Figure 8: Formation process of secondary upsetting defect (numerical simulation).

to increase of diameter, led to a decrease in temperature of the drum-shape area. At this moment, the materials which were newly fed into the galvanic area had not reached the forging temperature. Therefore, the newly entered materials were forced to deform at a relatively low temperature under the axial upsetting pressure and the inadequate deformation below the forging temperature had decreased the slope of the drum-shape, as shown in Figure 8 b. Subsequently, the location of the maximum-temperature area gradually drifted from the drum-shape area to the neck entrance area. Therefore, the drum-shape area stopped deformation, while the neck entrance area began to deform continuously. Meanwhile, the secondary upsetting began, and another drum-shape appeared, as shown in Figure 8c. Finally, as the second drum-shape grew up continuously, a deep groove between the two drum-shapes arose, as shown in Figure 8d.

\subsection{Optimization of processing parameters for secondary upsetting defect}

As analyzed above, the main factor that causes the secondary upsetting defect is the large decrease in temperature of the deformed portion after the formation of drum-shape. Therefore, the stability control of deformation temperature in the electric upsetting process can take effect against the secondary upsetting defect. It is commonly believed that the effect of astern speed of anvil and the upsetting pressure on the deformation temperature is very small in electric upsetting process. Consequently, the adjustment of astern speed of anvil and upsetting pressure can't prevent the workpiece from the secondary upsetting defect. Instead, small clamping length can increase the temperature in the neck entrance area in the preliminary stage of electric 


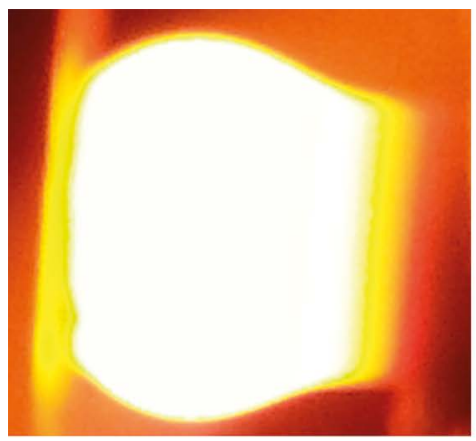

(a)

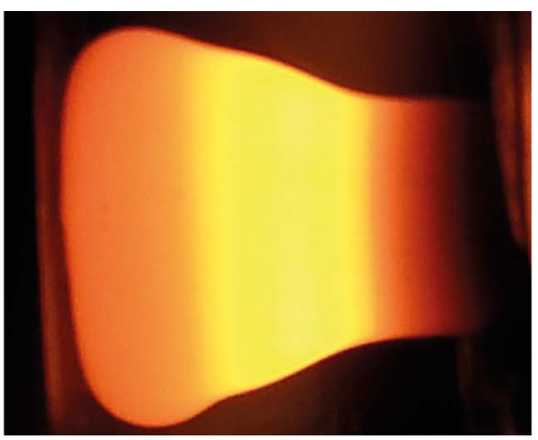

(b)

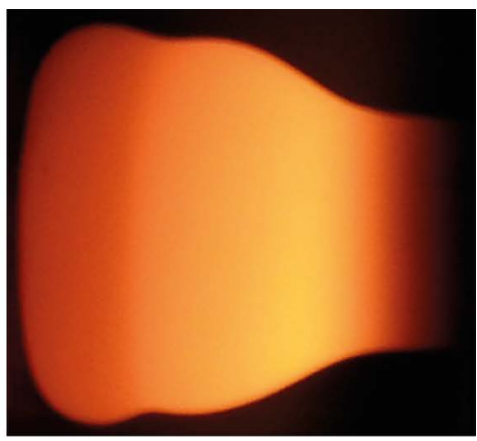

(c)

Figure 9: Formation process of secondary upsetting defect (actual production).

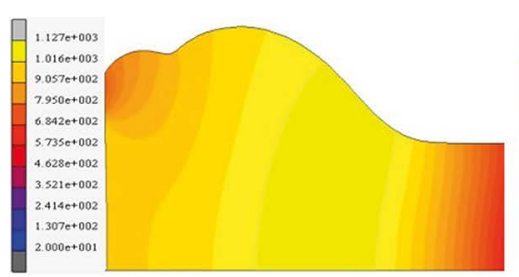

(a)

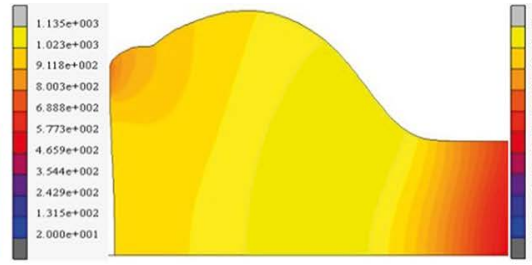

(b)

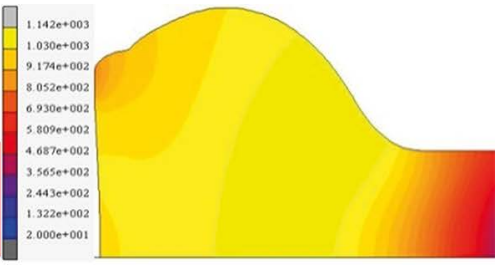

(c)

Figure 10: The shape of the workpiece after electric upsetting under different constant currents (a) $8042 \mathrm{~A}$, (b) $8595 \mathrm{~A}$, (c) $9550 \mathrm{~A}$.

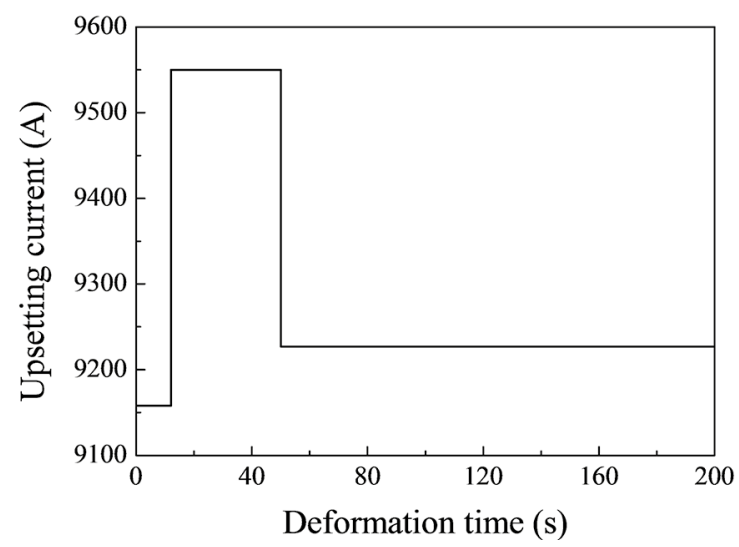

Figure 11: The three-stage current mode for electric upsetting of 3Cr20Ni10W2 heat-resistant alloy. upsetting, thus the secondary upsetting defect can be avoided. However, the temperature of the galvanic portion will be too high and the workpiece will be burnt if the clamping length is too small. As a result, the adjustment of clamping length is not available for the secondary upsetting defect.

In the electric upsetting process, the deformation temperature is mainly dependent on the upsetting current, since the workpiece is heated through the joule heat generated by the upsetting current. Therefore, the adjustment of upsetting current is the best approach to solve the problem of secondary upsetting defect. In order to increase the temperature of the deformed portion after the formation of drum-shape in the preliminary stage, higher upsetting currents were selected for the electric upsetting process. The other processing parameters were kept unchanged as in Table 3. Figure 10 shows the shape of the workpiece after electric upsetting under higher currents, in comparison with 8042 A. It can easily be found that the secondary upsetting defect becomes less distinct with the increase of upsetting current. However, the

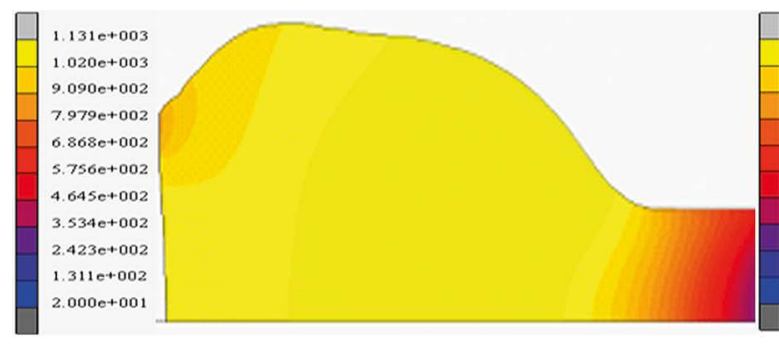

(a)

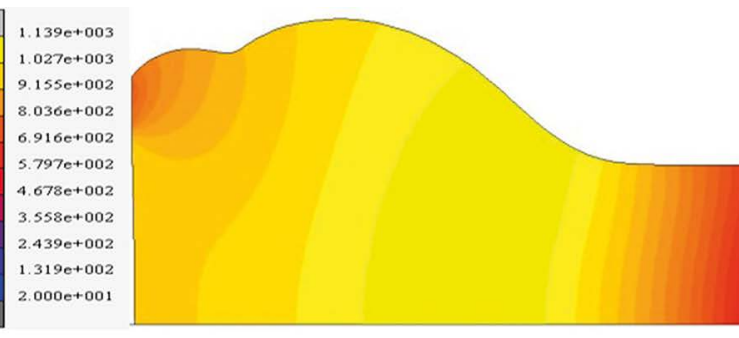

(b)

Figure 12: The forming results in numerical simulation: (a) under the three-stage current mode, (b) under the constant current mode. 


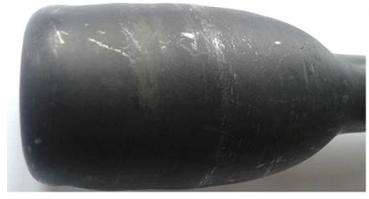

(a)

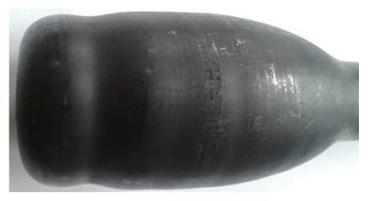

(b)
Figure 13: The forming results in experiment (a) under the threestage current mode, (b) under the constant current mode.

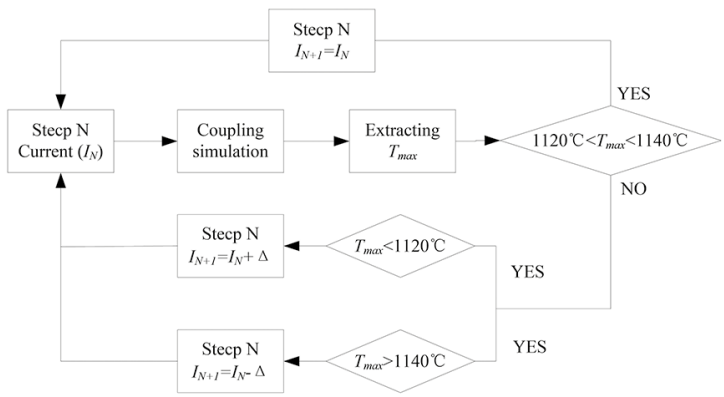

Figure 14: The algorithm of the current subroutine.

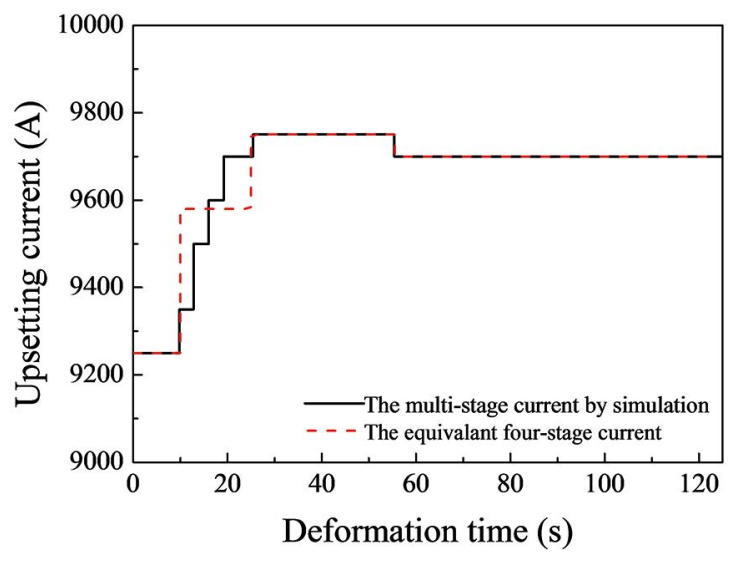

Figure 15: Simulated and equivalent multi-stage upsetting current.

secondary upsetting defect cannot be completely avoided under a constant current. Thus a three-stage current mode was put forward to eliminate the secondary upsetting defect. A relatively low current was selected in the preliminary stage of electric upsetting to ensure steady deformation. Subsequently, a current with high level was adopted to reduce the temperature decrease after the formation of the drum-shape. Finally, the current was set to a low level to avoid overheating. The three-stage current mode for electric upsetting of $3 \mathrm{Cr} 20 \mathrm{Ni} 10 \mathrm{~W} 2$ heat-resistant alloy is constructed through trial and errors, as shown in Figure 11. A numerical simulation of electric upsetting process under the three-stage current mode was conducted, in which the other processing parameters were kept unchanged as in Table 3 . The forming results in numerical simulation under the constructed three-stage current mode and the constant current mode are shown in Figure 12. It can be noticed that a smooth and symmetrical shape without secondary upsetting defect has been achieved under the three-stage current mode, in comparison with the grooved shape under constant current mode. In addition, the experimental comparison under the constructed three-stage current mode and the constant current mode has demonstrated that the constructed three-stage current mode is effective for the elimination of the secondary upsetting defect, as shown in Figure 13.

\subsection{An optimization method based on close-cycle control of current in numerical simulation}

The multi-stage current mode has been proved to be effective for the elimination of the secondary upsetting defect, as stated above. However, in terms of engine valves with different sizes and different materials, trial and error approach could be adopted, which may cost lots of time. Aiming at increasing the efficiency of current optimization, a novel optimization method based on close-cycle control of current in FE simulation was introduced. The principle of the optimization method is: (1) If the maximum temperature of the workpiece is higher than 1140 ${ }^{\circ} \mathrm{C}$ in the electric upsetting process, the current will be reduced at next step; (2) If the maximum temperature of the workpiece is lower than $1120{ }^{\circ} \mathrm{C}$, the current will be increased at next step. The optimization method was realized in current work by developing a subroutine in software MSC.Marc. The algorithm of the subroutine is shown in Figure 14. A numerical simulation of electric upsetting with the developed current subroutine was conducted, in which the other processing parameters remained the same as in Table 3. A seven-stage current mode was output from the software MSC.Marc after the simulation, and it was adjust to an equivalent four-stage current mode to match the electric upsetting machine, as shown in Figure 15. The forming result in numerical simulation with the developed subroutine is shown in Figure 16a, in comparison with the forming result in experiment under the equivalent four-stage current mode which

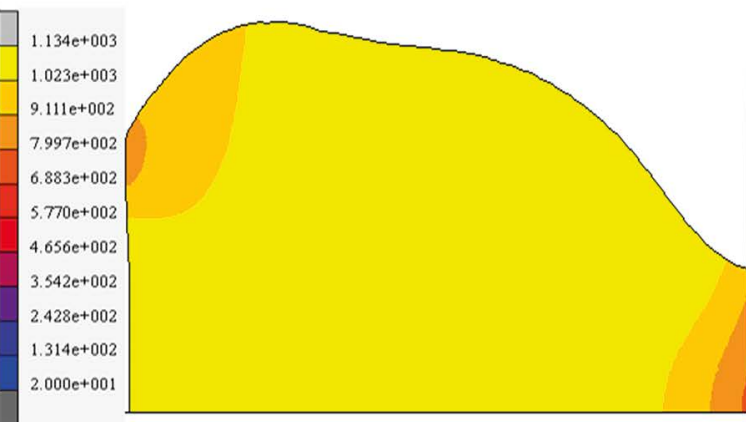

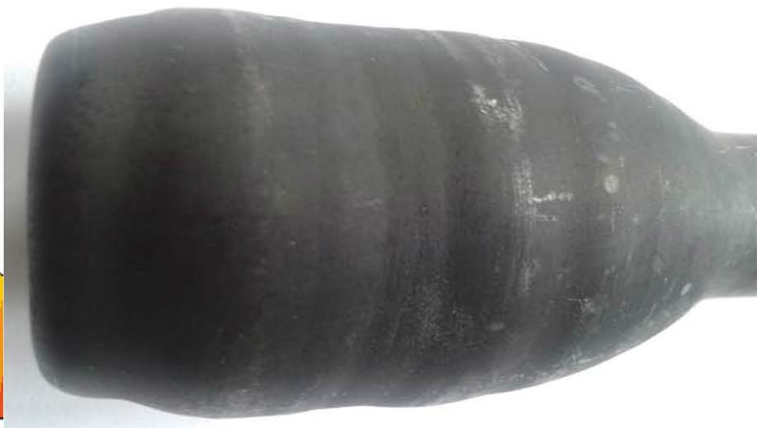

(b)

Figure 16: Forming results: (a) in numerical simulation with the developed subroutine, (b) in experiment under the equivalent four-stage current mode. 
is shown in Figure 16b. It is obviously been found in Figure 16 that drum-shape without defect was obtained, which has amply demonstrates the optimization is available in the elimination of secondary upsetting defect for electric upsetting process.

\section{Conclusions}

The formation process of the secondary upsetting defect in electric upsetting was analyzed based on the constructed 2D nonlinear electrical-thermal-mechanical multi-field coupling finite-element model, and corresponding optimization method was proposed. The following conclusion can be drawn:

(1) The main factor that causes the secondary upsetting defect is the large decrease in temperature of the deformed portion after the formation of drum-shape in the preliminary stage of electric upsetting with inappropriate combination of processing parameters.

(2) The secondary upsetting defect becomes less distinct with the increase of upsetting current, but it cannot be completely avoided by a constant current mode. However, the three-stage current mode is available to eliminate the secondary upsetting defect in electric upsetting process.

(3) A new optimization method based on close-cycle control of current in numerical simulation by developing a current subroutine in MSC.Marc was proved to be effective and efficient for the elimination of the secondary upsetting defect.

\section{Acknowledgements}

The work was supported by Open Fund Project of State Key Laboratory of Materials Processing and Die \& Mould Technology (No.P2014-16) and Chongqing foundation and advanced research project (cstc2016jcyjA0335).

\section{References}

1. Yanagimoto J, Izumi R. Continuous electric resistance heating-Hot forming system for high-alloy metals with poor workability. Journal of Materials Processing Technology. 2009;209(6):3060-3068.
2. Mori K, Maeno T, Fukui Y. Spline forming of ultra-high strength gear drum using resistance heating of side wall of cup. CIRP Annals - Manufacturing Technology. 2011;60(1):299-302.

3. Eggert GM, Dawson PR. Assessment of a thermoviscoplastic model of upset welding by comparison to experiment. International Journal of Mechanical Sciences. 1986;28(9):563-589.

4. Nied HA. The finite element modeling of the resistance spot welding process. Welding Journal. 1984;63(4):123s-132s.

5. Jeong HS, Cho JR, Lee NK, Park HC. Simulation of Electric Upsetting and Forging Process for Large Marine Diesel Engine Exhaust Valves. Eco-Materials Processing \& Design VII. 2006;510-511:142-145.

6. Wang YG, Yin XM. Analysis of parameters and microcomputer control for the electric upsetting process for forging engine valves. Journal of Materials Shaping Technology. 1987;5(2):125-131.

7. Sun Y, Liu T, Zhang Z, Zhang T, Luo T. Optimum control of process parameters in electrical upsetting. Proceeding of the Institute of Mechanical Engineers, Part B: Journal of Engineering Manufacture. 2003;217(9):1259-1263.

8. Kobayashi S, Oh SI, Altan T. Metal Forming and the FiniteElement Method (Oxford Series on Advanced Manufacturing). Oxford: Oxford University Press; 1989. 402p.

9. Sun X, Dong P. Analysis of aluminum resistance spot welding processes using coupled finite element procedures. Welding Journal. 2000;79(8):215s-221s.

10. Anzawa T, Yu Q, Yamagiwa M, Shibutani T, Shiratori M. Reliability evaluation on deterioration of power device using coupled electrical-thermal-mechanical analysis. Journal of Electronic Packaging. 2010;132(3):031012.

11. MSC Software. Marc 2010 (volume A): Theory and User Information. 2010 [Cited 2016 Jun 16]. Available from: https://simcompanion. mscsoftware.com/infocenter/index?page=content\&id=DOC9450

12. Saunders N, Guo Z, Li X, Miodownik AP, Schillé JP. Modelling the material properties and behaviour of Ni-based superalloys. Superalloys 2004. 2004;849-858.

13. Quan GZ, Luo GC, Wen HR. Influence of electric upsetting process variables on temperature field evolution by multi-field coupling finite element analysis. International Journal of Precision Engineering \& Manufacturing. 2015;16(7):1525-1531. 


\section{Erratum}

In the article "A Study on Formation Process of Secondary Upsetting Defect in Electric Upsetting and Optimization of Processing Parameters Based on Multi-Field Coupling FEM", DOI number: http://dx.doi.org/10.1590/1980-5373MR-2015-0678, published in Mat. Res., 19(4): 856-864, in the first page where it read:

Guo-zheng Quan ${ }^{\mathrm{a}, \mathrm{b} *}$, Zhen-yu Zou', Zhi-hua Zhang ${ }^{\mathrm{b}}, \mathrm{Jia}^{\mathrm{b}} \mathrm{Pan}^{\mathrm{b}}$

aState Key Laboratory of Materials Processing and Die \& Mould Technology, Huazhong University of Science and Technology, Hubei 430074, China

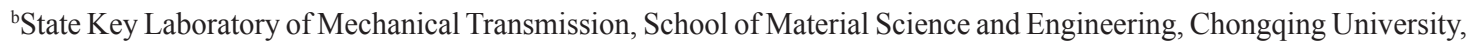
Chongqing 400044, China

It should be written:

Guo-zheng Quan, ${ }^{\mathrm{a}}$, Zhen-yu Zou', Zhi-hua Zhang ${ }^{\mathrm{b}}, \mathrm{Jia}^{\mathrm{b}} \mathrm{Pan}^{\mathrm{b}}$

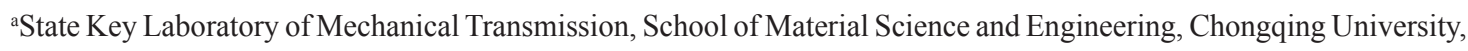
Chongqing 400044, China

${ }^{\mathrm{b} S t a t e}$ Key Laboratory of Materials Processing and Die \& Mould Technology, Huazhong University of Science and Technology, Hubei 430074, China

And in the page 864 where it read:

The work was supported by Open Fund Project of State Key Laboratory of Materials Processing and Die \& Mould Technology (No.P2014-16).

It should be written:

The work was supported by Open Fund Project of State Key Laboratory of Materials Processing and Die \& Mould Technology (No.P2014-16) and Chongqing foundation and advanced research project (cstc2016jcyjA0335). 